\title{
Electronic structure of zinc-blende MnTe within the GW ap- proximation
}

\author{
A. Fleszar*, M. Potthoff, and W. Hanke \\ Institut für Theoretische Physik und Astrophysik, Universität Würzburg, Am Hubland, 97074 Würzburg, \\ Germany
}

Key words Magnetic semiconductors, electronic structure.

PACS 71.55.Gs, 71.70.-d, 71.70.Gm

Using the local spin-density approximation (LSDA) and the (non self-consistent) GW approach, the (quasiparticle) band structure is calculated for MnTe in zinc-blende geometry. Different parameters characterizing the electronic structure are computed for an antiferromagnetic and the ferromagnetic phase and compared with the experiment. The strong Hubbard-type repulsion on the Mn-3d orbitals and the $p$ - $d$ hybridization are seen to be responsible for substantial defects found in the LSDA picture. It is discussed to which extent these can be improved upon by means of the GW approach.

Copyright line will be provided by the publisher

\section{Introduction}

Diluted magnetic semiconductors (DMS), i.e., semiconductors with a certain amount of diluted magnetic ions, are well-known materials for 30-40 years. The combination of semiconducting and magnetic phenomena in one and the same material gives rise to novel magnetic, magneto-optic, and magneto-transport properties which are interesting for technological applications as well as from fundamental, scientific point of view [1]. DMS materials have attracted a perhaps unprecedented world-wide interest after it was demonstrated that it might be possible to produce a ferromagnetic semiconductor at temperatures approaching room temperature [2]. The high solubility of Mn in II-VI compounds and the possibility to prepare goodquality samples with almost arbitrary composition range of $\mathrm{Mn}$ have made the $\mathrm{A}_{1-x}^{\mathrm{II}}-\mathrm{Mn}_{x}-\mathrm{B}^{\mathrm{VI}}$ ternary compounds the basic representatives of the DMS material class. $\mathrm{Cd}_{1-x} \mathrm{Mn}_{x} \mathrm{Te}$ and $\mathrm{Zn}_{1-x} \mathrm{Mn}_{x} \mathrm{Te}$ are perhaps the most studied members of this group.

In a phenomenological approach to the magnetic properties of DMS materials, one usually describes the Mn-Mn interactions with a Heisenberg model, while the interaction of band electrons or holes with the localized Mn magnetic moments is modelled by means of a Kondo Hamiltonian [1]. The parameters of these models, i.e., the exchange constants of the Heisenberg model, and the $J_{s p-d}$ parameters of the Kondo Hamiltonian, have to be interpreted as effective parameters that, via (strong-coupling) perturbation theory, can be traced back to the Hubbard- $U$ repulsion on the Mn-3d shell and the relative position of the Mn-3d states with respect to the valence-band maximum (VBM) [3]. In any case, phenomenological or ab initio, the electronic and magnetic properties of DMS materials are determined in first place by the electronic configuration of the substitutional $\mathrm{Mn}$ ion and its interaction with the surrounding anions.

Mn formally belongs to the group VII of the periodic table. Its atomic valency is given by five $3 d$ and two $4 s$ electrons. Substituted as a cation in a II-VI compound, however, Mn behaves as a group II element. The two $4 s$ electrons build bonds together with the anions' valence $p$ electrons, while the $3 d$ electrons remain in their atomic $S=\frac{5}{2}$ high-spin configuration giving rise to a localized magnetic moment within the semiconducting host. Apart from its magnetic moment, the half-filled $3 d$ shell of Mn

* Corresponding author: e-mail: fleszar@physik.uni-wuerzburg.de, Phone: +49931 888 5879, Fax: +49 9318885141 
resembles the fully filled $d$ shell of $\mathrm{Zn}, \mathrm{Cd}$, or $\mathrm{Hg}$ in II-VI compounds. The interaction of the valence $s p$ electrons with the localized $d$ electrons has an important effect on the properties of these materials. It considerably complicates the ab initio description of II-VI semiconductors since an independent-particle picture becomes problematic. At least with respect to excitation properties, this makes calculations based on the local-density approximation (LDA) [4] questionable. Furthermore, the LDA is known to strongly overestimate the $s p$ - $d$ hybridization, thus pushing up the VBM and making the fundamental energy gap much smaller than it would result from the "usual" neglect of many-body effects in LDA for standard semiconductors [5].

In the case of the substitutional Mn in II-VI compounds as well as for pure Mn-chalcogenides, there are two factors that are relevant in the context of $s p$ - $d$ interaction and that are novel when comparing with the normal II-VI materials. First, the energy position of the atomic $\mathrm{Mn}-3 d$ level is at least $3 \mathrm{eV}$ higher (with respect to the vacuum level) than the positions of the $3 d, 4 d$ and $5 d$ levels of $\mathrm{Zn}, \mathrm{Cd}$ and $\mathrm{Hg}$, respectively. As a result, the Mn- $d$-shell-derived bands appear in II-VI DMS materials or in pure Mn-chalcogenides at about 3.5-4 eV below the VBM, i.e., they are resonant with the $s p$ bands. This is a new feature compared with II-VI compounds where the $d$-shell-derived bands are situated at 10-8 eV below VBM and are therefore well separated from the $s p$ bands. Second, the spin configuration of the Mn ions is also relevant for the hybridization because of the Hubbard- $U$ "energy barrier" for the spin-down (unoccupied) states. In the normal II-VI compounds the spin-up and spin-down states are both occupied and have the same energy position.

In this paper we discuss the band structure of zinc-blende MnTe as a prototype material. Results obtained with the conventional local spin-density approximation (LSDA) [4, 6] are presented and contrasted with many-body corrections obtained from the GW approach [7, 8]. The technique used is similar to our earlier studies of the electronic structure of the II-VI compounds [9] and other semiconductors [10, 11]. A comparison with the case of non-magnetic II-VI materials is one of the goals of this paper. The band structures of MnTe in different magnetic phases are analyzed and some phenomenological parameters are determined.

\section{Zinc-blende MnTe}

In nature, MnTe crystallizes in the hexagonal NiAs structure. It is an antiferromagnet below a Néel temperature of $307 \mathrm{~K}$. The zinc-blende phase (ZB) of MnTe is metastable. It is easily grown, however, with the MBE technique. Hence, ZB MnTe bas intensively been studied for about 18 years and is oftenly used as a magnetic component in Mn-based diluted magnetic semiconductors. Apart from technological interest, ZB MnTe is also a fundamentally interesting material as it represents one of the few realizations of fcc antiferromagnetic systems with dominant nearest-neighbor interactions resulting in an inherent magnetic frustration. Upon cooling the system usually undergoes a structural distortion that accompanies the magnetic phase transition which eventually lifts the ground-state degeneracy. This also happens with ZB MnTe which developes a tetragonal distortion of the order of $0.3 \%$ below the Néel temperature [12].

Zinc-blende MnTe is a type-III antiferromagnet (AF3) with a Néel temperature of $65 \mathrm{~K}$ [13]. The difference to the simpler type-I structure (AF1) constists in the different geometrical sequence of the (001) planes with the planar antiferromagnetic order: In the AF1 structure this is given by an A-B-A-B-A sequence of (001) planes, while the AF3 phase has a twice as long period: A-B-C-D-A. The ferromagnetic phase has higher energy and is not observed experimentally. Antiferromagnetic order is obviously favored by the superexchange mechanism [14], dominant in the intrinsic Mn-chalcogenides as well as in all II-MnVI DMS materials. Another important experimental fact is the negative, i.e., antiferromagnetic coupling of the spins of (itinerant) holes with the localized spins of Mn [1]. This $J_{p d}$ coupling is very large in magnitude and the source of many characteristic properties of the DMS materials.

Cubic MnTe has been investigated experimentally with various techniques and especially its magnetic [13, 12] and elastic [15] properties are well characterized. In the present context, it is important to mention 
that the photoemission experiments place the occupied Mn-3d level at about $3.5 \mathrm{eV}$ below the VBM [16 [17] whereas the experimental exchange splitting of Mn-3d levels is about $6.9 \mathrm{eV}$ [18]. Theoretically, ZB MnTe was first studied within the LSDA approximation in two seminal papers by Wei and Zunger [19] and Larson et al [3]. Heisenberg exchange constants for the three Mn-chalcogenides (MnS, MnSe and $\mathrm{MnTe}$ ) have been derived from total-energy LSDA calculations (and empirically modified LSDA) by Wei and Zunger [20]. In addition, structural properties of MnTe have been studied within LDA and the generalized-gradient approximation [21].

\section{Computational details}

The first part of calculations presented here has been done within the LSDA approximation and the KohnSham approach of density-functional theory [4]. We use the Perdew-Zunger parameterization of the LDA functional [22] together with the interpolation formula of von Barth and Hedin [6] for intermediate spin polarization. Within the pseudopotential approach, a $\mathrm{Mn}^{15+}$ pseudopotential of the Hamann type [23] has been generated. Thus the whole $n=3$ atomic shell of $\mathrm{Mn}$ is taken into account in the self-consistent calculation, i.e., including the $3 \mathrm{~s}$ and $3 \mathrm{p}$ core states. For the tellurium atom a $\mathrm{Te}^{6+}$ pseudopotential of the BHS-type has been used [24]. The explicit inclusion of the Mn 3s and 3p states in calculations for solids is necessary for the subsequent $\mathrm{GW}$ calculation because of the large bare exchange interaction within the whole $n=3$ shell [25]. On the level of the LSDA approximation, however, using a $\mathrm{Mn}^{7+}$ pseudopotential results in basically the same band structure as the $\mathrm{Mn}^{15+}$ pseudopotential considered here - provided that the charge density of inner core shells is included in form of a partial core-charge correction [26].

The Kohn-Sham equations have been solved using the mixed-basis method. In addition to plane waves, a number of localized Gaussians multiplied by the $s, p$ and $d$ spherical harmonics are placed in the positions of the Mn atoms. Using this mixed basis has two advantages which are particularly important within the context of GW calculations: (i) The basis is universal enough to describe both, the strongly localized core states as well as the highly delocalized excited states which both are needed in the course of a GW calculation. (ii) As compared to a pure plane-wave scheme, it allows to considerably reduce the number of plane waves.

The GW method has been applied in a similar way as in our recent calculation for II-VI compounds [9]. The dynamical dielectric matrices are determined on the $(4,4,4)$ Monkhorst and Pack mesh of k-points [27], no plasmon-pole approximation has been applied. The dielectric matrices are calculated in Fourier space, and only the diagonal contribution of the self-energy has been taken into account [9]. The calculations

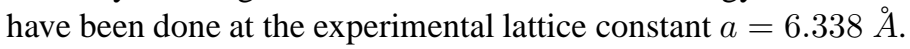

\section{Results of LSDA calculations}

Figure 1 shows the resulting LSDA band structure of zinc-blende MnTe for the ferromagnetic and the AF1 antiferromagnetic phase. Both magnetic structures do not appear in nature. Nevertheless, their analysis is highly instructive for the discussion of the Mn- $3 d-\mathrm{Te}-5 p$ hybridization. Note that the AF1 structure is very similar to the (actually realized) AF3 structure as regards the densitiy of states, for example. Considering the AF1 structure, however, allows for a more transparent discussion and facilitates the analysis below. The band structures shown in Fig. 1 agree well with previous LSDA results [19].

As can be seen from Fig. 1, ferromagnetic ZB MnTe appears to be a metal within the LSDA approximation. The "unoccupied" minority-spin bands drop down below the VBM in the majority-spin channel. By contrast, in the AF1 phase ZB MnTe turns out to be a semiconductor with a band direct gap at $\Gamma$ of 1.37 $\mathrm{eV}$ and a minimum indirect gap of $1.28 \mathrm{eV}$ at $X$. We have also calculated the band structure for the AF3 phase. In this case the direct band gap at $\Gamma$ is the minimum gap which amounts to $1.33 \mathrm{eV}$. These LSDA gaps are considerably smaller than the experimentally determined gap of $3.2 \mathrm{eV}[28,29$, 30]. This should be interpreted as the usual (and joint) artifact of Kohn-Sham theory and LDA approximation. 

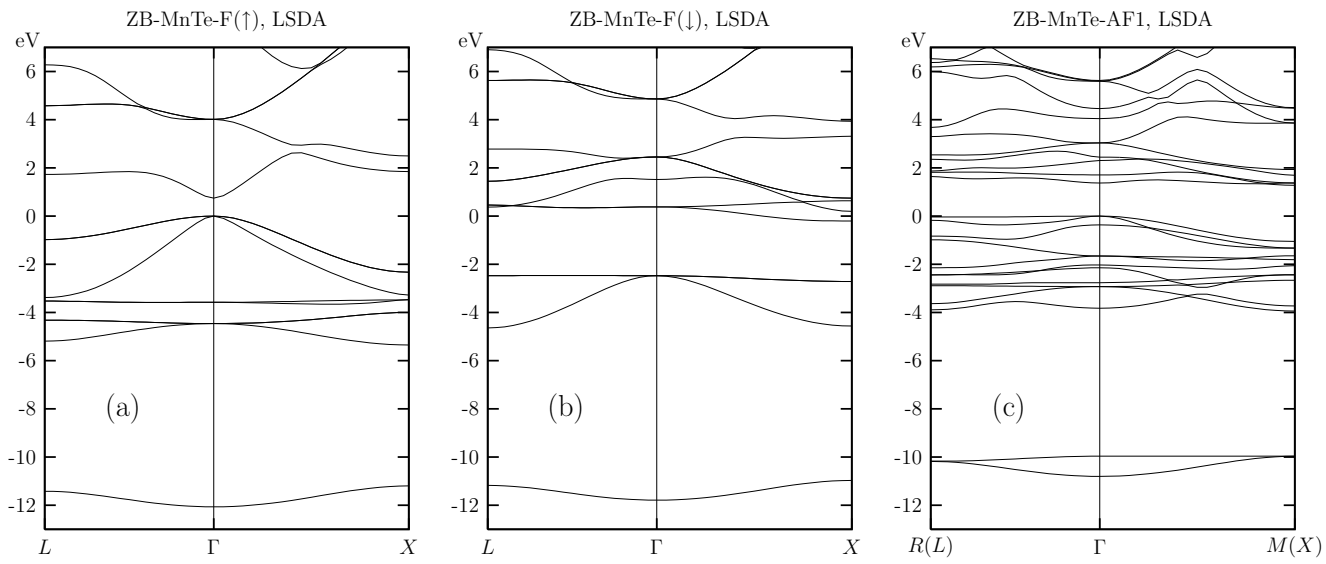

Fig. 1 LSDA band structure of zinc-blende MnTe in the ferromagnetic phase: (a) majority spin, (b) minority spin. Results for the antiferromagnetic AF1 phase: (c).

The spin-dependent density of states (DOS) for the ferromagnetic phase is presented in Fig. 2 (upper panel). The energy zero is placed at the VBM in the majority-spin channel. The dotted line shows the position of the Fermi energy. The lower panel presents the contributions to the DOS from the Mn- $3 d$ orbitals. Projections onto states with $\mathrm{e}_{g}$ and $\mathrm{t}_{2 g}$ symmetries are shown separately. In Fig. 3 the DOS for the $\mathrm{AF} 1$ phase is presented with a separate projection onto two inequivalent Mn atoms in the AF1 unit cell.

There are a few features worth commenting:

(i) In the ferromagnetic phase, the VBM of the minority-spin carriers is situated $2.47 \mathrm{eV}$ below the VBM in the majority-spin channel. This negative (and large) spin splitting of the VBM is common to all II-Mn-VI DMS materials and due to the negative (antiferromagnetic) $p$ - $d$ exchange interaction. This effective interaction can easily be understood as resulting from the Schrieffer-Wolff transformation [31] applied to Anderson's $p$ - $d$ model Hamiltonian [32]. The negative spin splitting of the VBM is therefore to be interpreted as a consequence of the strong Mn- $3 d$ Hubbard- $U$ repulsion and the fact that the $p$ bands lie above the occupied but below the unoccupied Mn-3d levels.

The $p$ - $d$ exchange is usually described by the phenomenological parameter $N_{0} \beta$ [1] defined by the relation: $E_{\mathrm{VBM}}(\uparrow)-E_{\mathrm{VBM}}(\downarrow)=-N_{0} \beta x\left\langle S_{z}\right\rangle$, where $x$ is the concentration of the magnetic ions $(x=1$ in our case) and $\left\langle S_{z}\right\rangle$ is the average value of the total spin on the magnetic ion. It is difficult to give a precise definition of the magnetic moment of a single atom embedded in a crystal. For the ferromagnetic phase $\left\langle S_{z}\right\rangle=5 / 2$ in the whole unit cell resulting in a moment of $5 \mu_{\mathrm{B}}$ (we ignore that the magnetization actually slightly differs from $5 \mu_{\mathrm{B}}$ within a band calculation for a metal). The magnetic moment on the Mn-ion, however, is less than $5 \mu_{\mathrm{B}}$ due to the hybridization. In our calculation, the net magnetic moment in a sphere of radius $R_{M T}=1.37 \AA$ (half of the distance to the next Te-neighbor) is $4.5 \mu_{\mathrm{B}}$ for the ferromagnetic phase and $4.3 \mu_{\mathrm{B}}$ for the AF1 phase. Thus, taking $\left\langle S_{z}\right\rangle=4.5 / 2$ one obtains an LSDA value of $N_{0} \beta=-1.1 \mathrm{eV}$. The experimental value for ZB MnTe is $N_{0} \beta^{\exp }=-0.88 \mathrm{eV}$ [33]. This large magnitude of $N_{0} \beta$ results from a strong $p-d$ hybridization between Mn- $3 d$ and the anion- $p$ states. Within LSDA the hybridization strength is overestimated [5, 9]. This explains the larger theoretical value for $N_{0} \beta$ as compared to the experiment.

(ii) The exchange splitting of the unoccupied $4 s$ states at the $\Gamma$ point is positive. In Fig. 1 the conduction-

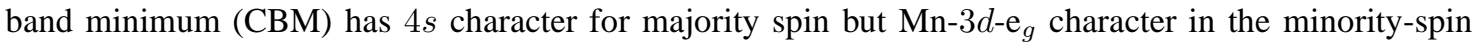
channel. For minority spin, the unoccupied $4 s$ states lie above Mn- $3 d-\mathrm{e}_{g}$ states at $\Gamma$. Hence, the energy difference between the $s$-type bands for spin up and down is recognized to be positive and amounts to $0.77 \mathrm{eV}$ in our calculation. This gives rise to $N_{0} \alpha=0.34 \mathrm{eV}$ to be compared with the experimental value of $N_{0} \alpha^{\exp }=0.22 \mathrm{eV}$ [33] $\left(N_{0} \alpha\right.$ is defined via the relation: $\left.E_{4 s}(\uparrow)-E_{4 s}(\downarrow)=-N_{0} \alpha x\left\langle S_{z}\right\rangle\right)$. 

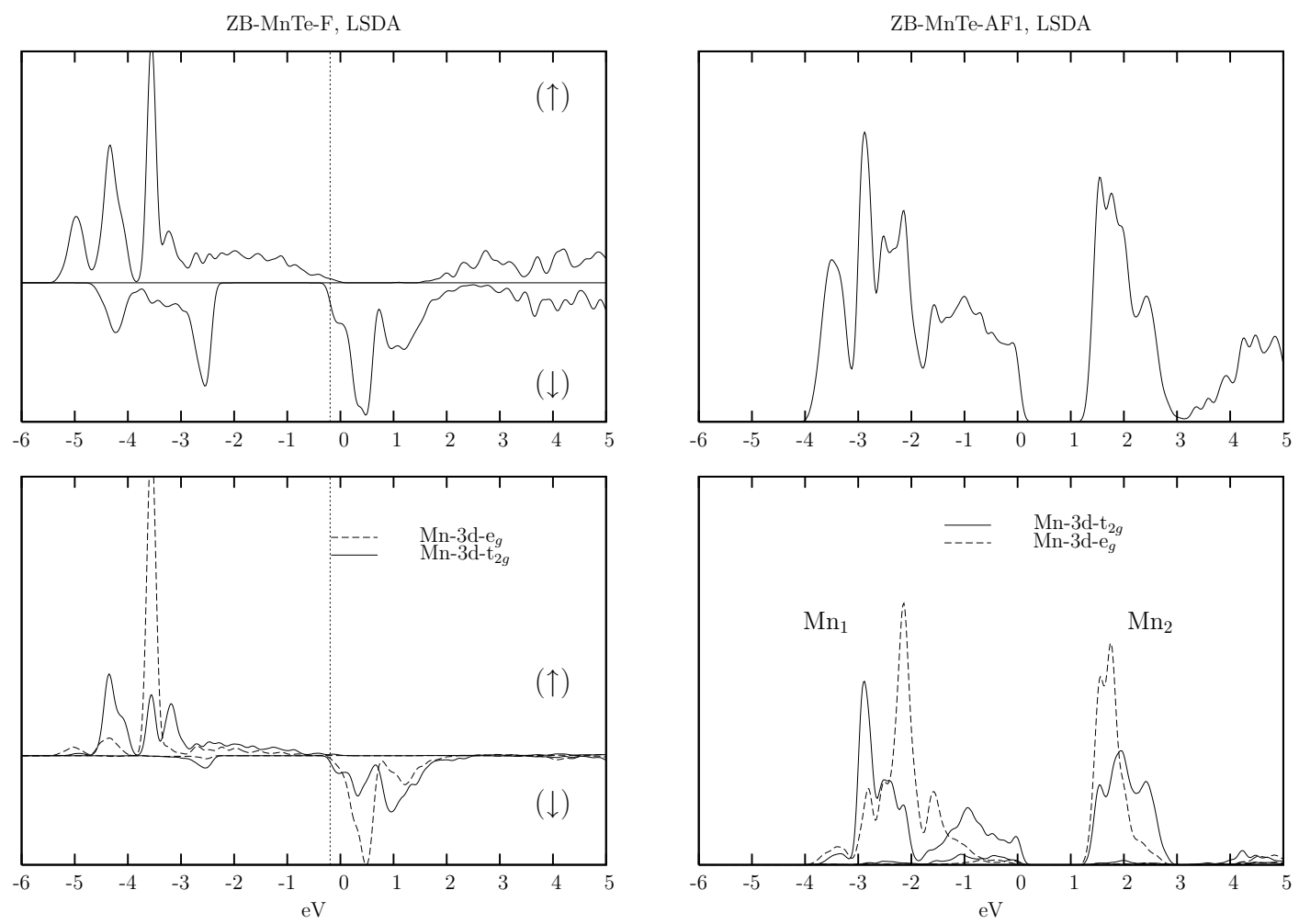

Fig. 2 LSDA density of states (DOS) for ZB MnTe in the ferromagnetic phase. Upper panel: total DOS. Lower panel: contributions from the Mn- $3 d-\mathrm{e}_{g}$ and Mn- $3 d-\mathrm{t}_{2 g}$ states. $(\uparrow)$ denotes the majority-spin, $(\downarrow)$ the minority-spin channel. Energy zero: valenceband maximum for spin $(\uparrow)$. Dotted line: Fermi en-

Fig. 3 LSDA DOS for ZB MnTe in the AF1 phase. Upper panel: total DOS. Lower panel: contributions from the Mn- $3 d-\mathrm{e}_{g}$ and $\mathrm{Mn}-3 d-\mathrm{t}_{2 g}$ states. The DOS is shown for two inequivalent $\mathrm{Mn}$ atoms in the AF1 unit cell. ergy

A positive value of $N_{0} \alpha$ indicates that for the $4 s$ states the spin splitting mechanism is of a direct (or potential) type. Note that at $\Gamma$ the $\mathrm{Mn}-4 s$ wave function is orthogonal to the $\mathrm{Mn}-3 d$ wave functions and has a large amplitude at the $\mathrm{Mn}$ sites. These are conditions that favour a parallel spin configuration according to Hund's rule.

(iii) Another characteristic feature is the decreasing width of the occupied $p$ - $d$-type bands when going from the majority-spin channel in the ferromagnetic phase $(\mathrm{F}(\uparrow))$, to the AF1 phase, and eventually to the minority-spin channel in the ferromagnetic phase $(\mathrm{F}(\downarrow))$. This is clearly seen in the plots of the density of states. The widths are $5.4 \mathrm{eV}, 3.9 \mathrm{eV}$ and $2.2 \mathrm{eV}$ for the $\mathrm{F}(\uparrow), \mathrm{AF} 1$ and $\mathrm{F}(\downarrow)$ case, respectively. The trend is caused by the decreasing number of hybridization partners for the Te- $5 p$ states within this series. While for the $\mathrm{F}(\uparrow)$ case each Te atom has four Mn-3d partners of spin up in the local environment with tetrahedral symmetry, there are only two such partners for the AF1 case. On the other two Mn atoms (with spin-down magnetic moment) the spin-up $3 d$ orbitals, which could hybridize, are separated in energy by the Hubbard- $U$, and therefore hybridization is largely suppressed. In the case of $\mathrm{F}(\downarrow)$, all Mn-3d orbitals of the same spin are separated by the Hubbard- $U$ energy barrier. Consequently, this gives the narrowest band. When extracting a quantitative value for the strength of the $p$ - $d$ hybridization, which is needed for the construction of an $s p$ - $d$ model Hamiltonian, one should be aware, however, that the LDA overestimates the hybridization strength. The size of this "LDA error" is difficult to estimate. 
Fleszar, Potthoff, and Hanke: ZB MnTe in the GW approximation

(iv) A feature related to the band widths is the position of the Mn- $3 d$ states. The lower panels of Figs. 2 and 3 show the DOS projected onto the Mn-3d contributions and resolved according to $\mathrm{e}_{g}$ and $\mathrm{t}_{2 g}$ symmetry. For the ferromagnetic phase, not only the crystal lattice but also the Kohn-Sham potential is characterized by the zinc-blende (Td) symmetry. In this case, the Mn-3d states with $\mathrm{e}_{g}$ symmetry do not hybridize with the Te- $5 p$ states, at least in the region close to the $\Gamma$ point. Therefore, one observes a rather narrow peak at $-3.6 \mathrm{eV}$ (see dashed line in the lower panel of Fig. 2). By contrast, the Mn-3d states with $\mathrm{t}_{2 g}$ symmetry strongly mix with the Te- $5 p$ states resulting in a comparatively broad partial DOS with a few peaks and ranging up to the VBM. For zinc-blende MnTe in the AF1 phase, the symmetry of the Kohn-Sham potential is lower than Td (tetragonal magnetic symmetry). Therefore, the contribution from the $\mathrm{e}_{g}$ states to the total DOS (dashed line in the negative-energy part of Fig. 3) is visibly broader than for the $\mathrm{F}(\uparrow)$ case and composed of a few peaks around the central peak at $2.1 \mathrm{eV}$ below the VBM. Hence, the difference between $-3.6 \mathrm{eV}$ for the $\mathrm{F}(\uparrow)$ case and $-2.1 \mathrm{eV}$ for the AF1 case can be explained with the different available number of hybridization partners with the same spin. One should note, however, that this energy difference, $1.5 \mathrm{eV}$, is perhaps to a large extent due to the considerable overestimation of the $p$ - $d$ hybridization strength within the LDA. One could speculate that in a theory free from the LDA errors, the position of the $\mathrm{e}_{g}$ peak could be almost independent of the magnetic phase.

(v) The exchange splitting of the Mn- $3 d$ states is not straightforward to define because the Mn- $3 d$ states undergo crystal-field and hybridization-induced splittings into several bands. We will define two exchange splittings, separately for $\mathrm{e}_{g}$ and $\mathrm{t}_{2 g}$ symmetry, by calculating the center of mass of the symmetry-projected DOS. Furthermore, we ignore the hybridization effects and limit the considerations to peaks below $-2 \mathrm{eV}$ for the AF1 structure and $\mathrm{t}_{2 g}$ symmetry and below $-2.9 \mathrm{eV}$ for the $\mathrm{F}(\uparrow)$ case and the same symmetry. With these definitions we obtain the values $\Delta_{e_{g}}=4.3 \mathrm{eV}$ and $\Delta_{t_{2 g}}=4.8 \mathrm{eV}$ in the ferromagnetic phase and $\Delta_{e_{g}}=3.9 \mathrm{eV}$ and $\Delta_{t_{2 g}}=4.7 \mathrm{eV}$ in the $\mathrm{AF} 1$ phase. We note that the exchange splittings for the ferromagnetic phase are larger than for the AF1 phase as was already noticed in [19] but the effect is small. This reflects the local nature of this splitting having its origin in the Hubbard- $U$ on the Mn- $3 d$ shell.

\section{Results of GW calculations}

Our main interest concerns the changes in the LDA band structure that are introduced by the GW selfenergy. The difference between the LDA Kohn-Sham potential and the GW self-energy comes from the difference between the LDA exchange-correlation potential and the sum of the bare exchange and the dynamically screened Coulomb potential of an extra particle in the GW approximation. In perturbative GW theory, as it is used here, the LDA wave functions remain unchanged. This implies that the $p$ - $d$ hybridization is unchanged, i.e., an important ingredient affecting the band structure for all $\mathrm{II}^{B}$-VI compounds. We conclude that, for this situation, GW corrections carry information about the effects of screening and the effects of the true exchange, not properly included in the LDA theory, but do not correct the LDA errors with respect to the hybridization. In addition, the screening as well as the bare exchange are calculated on the basis of the LDA band structure, i.e., they are not free of LDA errors. A remedy of these problems would be an application of the GW method either in a self-consistent way, or on top of a one-particle approximation free from the most severe LDA errors. It is nevertheless instructive to see the effects of the bare exchange and screening and their influence on the electronic structure in different magnetic phases.

Fig. 4 presents the band structure of ZB MnTe in the ferromagnetic and in the AF1 phase as obtained within the GW approximation. Figs. 5 and 6 show the corresponding densities of states. They appear somewhat smoother as compared to Figs. 3 and 4. This is due to the slightly larger energy broadening and the coarser mesh of k-points on which the GW density of states has been calculated.

The most apparent effect of the GW correction consists in an opening of the energy gap. Opposed to the LSDA result, ferromagnetic ZB MnTe in no longer a metal but exhibits an overall positive energy gap. The majority-spin energy gap at $\Gamma$ is $1.71 \mathrm{eV}(0.74 \mathrm{eV}$ in the LSDA). The gap for the minority-spin case, as measured between the $\Gamma_{15 v}(\downarrow)$ and $\Gamma_{1 c}(\downarrow)$ states, has increased from $4 \mathrm{eV}$ in the LSDA to $4.5 \mathrm{eV}$ in the 

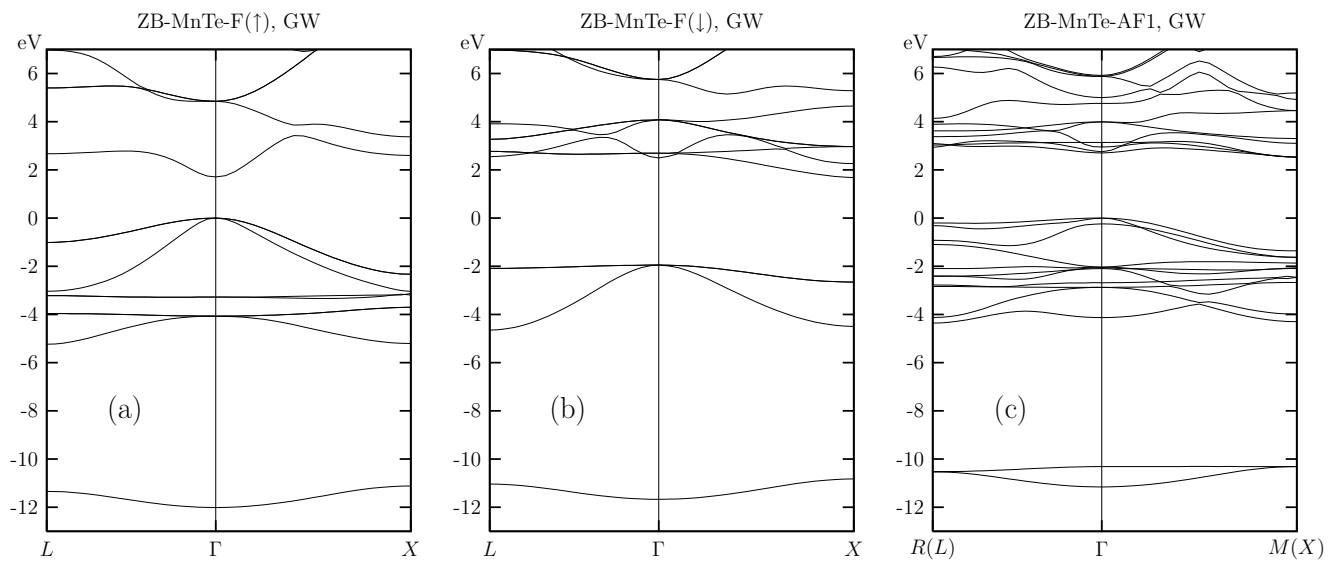

Fig. 4 GW band structure of zinc-blende MnTe in the ferromagnetic phase. (a) majority spin, (b) minority spin. (c) Band structure for the antiferromagnetic AF1 phase.

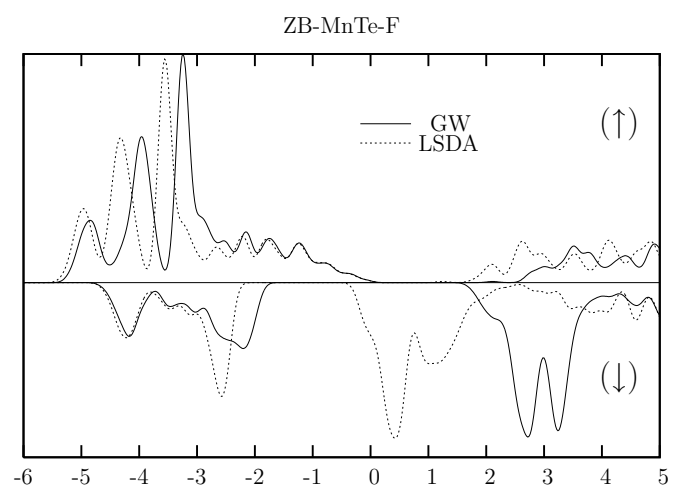

Fig. 5 DOS for ferromagnetic ZB MnTe. Solid line: GW, dotted line: LSDA.

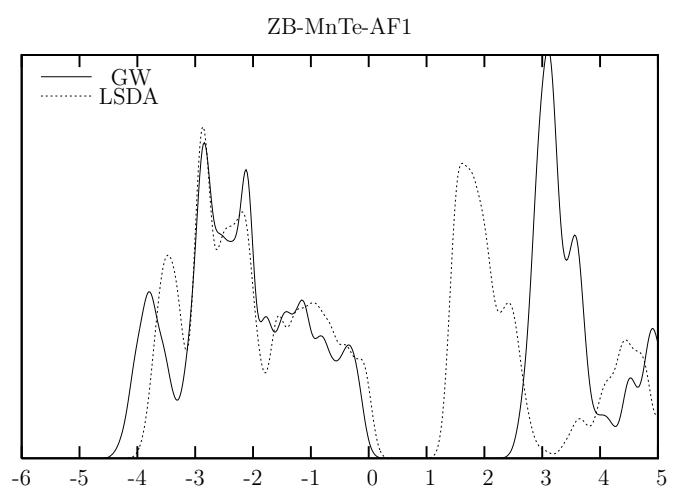

Fig. 6 DOS for antiferromagnetic (AF1) ZB MnTe. Solid line: GW, dotted line: LSDA.

GW approach. For the AF1 phase, the GW energy gap turns out to be $2.7 \mathrm{eV}$ at $\Gamma$ and $2.53 \mathrm{eV}$ at $X$, to be compared with the LSDA gap of $1.37 \mathrm{eV}$ at $\Gamma$ and $1.28 \mathrm{eV}$ at $X$. Similarly as in the LSDA, the lowest conduction band has a Mn- $3 d$ character. The $s$-type conduction band, however, has only $0.1 \mathrm{eV}$ higher energy. Assuming that the spin-orbit (SO) splitting of the VBM is similar in MnTe and other tellurides, the energy gap would be $\sim 0.3 \mathrm{eV}$ smaller with $\mathrm{SO}$ effects included. Hence, it would be $\sim 0.8 \mathrm{eV}$ smaller than the experimental gap in the $\mathrm{AF} 3$ phase which amounts to $3.2 \mathrm{eV}$. This discrepancy is twice as large as for ZnTe and CdTe where GW theory predicts an $0.4 \mathrm{eV}$ smaller gap compared with the experiment [9]. It is reasonable to ascribe this difference to the stronger hybridization effects in MnTe as compared to $\mathrm{II}^{B}$-VI compounds.

As can be seen in Figs. 5 and 6, the largest GW corrections show up for the unoccupied Mn-3d states. The spin-down Mn- $3 d$ states in the ferromagnetic phase are shifted by more than $2 \mathrm{eV}$ to higher energies. The unoccupied Mn- $3 d$ states in the AF1 phase are shifted by $1.5 \mathrm{eV}$. These corrections are larger than those for diffuse $s p$ bands and can be understood as a cancellation of a considerable LDA error.

The occupied Mn-3d states either retain their LDA energy position relative the VBM, as it is the case for the $\mathrm{AF} 1$ phase, or shift by $\sim 0.3 \mathrm{eV}$ to higher energies as for the ferromagnetic phase. This is a surprising result because one could rather expect an (opposite) downshift of the localized $d$ states which are underbound within the LDA. For $\mathrm{Zn}$ - and Cd-chalcogenides the cations' $d$ states are known to shift down by about $0.5-0.7 \mathrm{eV}$ due to the $\mathrm{GW}$ correction [9]. This shift is not sufficient to achieve agreement 
with the experiment, and one of the reasons for the remaining discrepancy is the LDA starting point of the $\mathrm{GW}$ procedure. We believe that this is the same reason why the Mn-3d states in the present calculation do not move or move upwards, respectively. For MnTe the $p$ - $d$ hybridization is stronger than for $\mathrm{II}^{B}$-VI materials. This stronger hybridization precludes a downshift of the Mn-3d states in the AF1 phase and, for the ferromagnetic phase where there are even more hybridization partners, results in an upwards shift. Since GW theory tends to decrease the width of $p$-valence bands in II $^{B}$-VI materials [9], an admixture of $p$-type wave functions to $3 d$-type states gives rise to a competition between down and up GW shifts.

The average energy position of the Mn- $3 d$ occupied states for the AF1 phase is the same within LSDA and $\mathrm{GW}$ and equal to $2.5 \mathrm{eV}$ below the VBM. This agrees well with the results of Wei and Zunger [19]. In photoemission experiments [16, 17] only one Mn peak was resolved at $3.5 \mathrm{eV}$ below VBM.

The GW exchange splittings of Mn-3d states in the AF1 phase, $\Delta_{e_{g}}=5.4 \mathrm{eV}$ and $\Delta_{t_{2 g}}=6.2 \mathrm{eV}$, should be compared with the experimental value of $6.9 \mathrm{eV}$ [18]. For the ferromagnetic phase, $\mathrm{GW}$ predicts $\Delta_{e_{g}}=6.2 \mathrm{eV}$ and $\Delta_{t_{2 g}}=6.7 \mathrm{eV}$, respectively. Since within $\mathrm{GW}$ the energies of quasiparticle states can be interpreted as total-energy differences of systems with $N$ and $N \pm 1$ particles, the calculated exchange splittings can be seen as the GW approximation for the screened Hubbard- $U$.

The GW exchange splitting of the VBM in the ferromagnetic phase of $-1.95 \mathrm{eV}$ is smaller than in the LDA. This results in $N_{0} \beta=-0.87 \mathrm{eV}$ within the GW approximation which is very close to the experimental value of $-0.88 \mathrm{eV}$. For the exchange splitting of the $s$-type conduction bands at $\Gamma$, the $\mathrm{GW}$ calculation yields $E_{4 s}(\uparrow)-E_{4 s}(\downarrow)=-0.79 \mathrm{eV}$. This gives rise to $N_{0} \alpha=0.35 \mathrm{eV}$ which, similarly as in case of the LSDA, is larger than the experimental value $N_{0} \alpha^{E x p}=0.22 \mathrm{eV}$. One should remember, however, that the theoretical results suffer from the uncertainty in the definition of the local magnetic moment on the Mn ion while the experimental results have been determined as a statistical average on systems with different Mn content.

Tables 1 and 2 collect the values of the parameters derived in this work in comparison with previous calculations as well as with the experiment.

Table 1 Energy levels and exchange splittings for $\mathrm{ZB}-\mathrm{MnTe}$ in the F phase. All values in $\mathrm{eV}$.

\begin{tabular}{lrr}
\hline & LDA & GW \\
\hline$\Gamma_{15 v}(\uparrow)-\Gamma_{15 v}(\downarrow)$ & 2.47 & 1.95 \\
$\Gamma_{1 c}(\uparrow)-\Gamma_{1 c}(\downarrow)$ & -0.77 & -0.79 \\
$E_{g}(\uparrow): \Gamma_{1 c}^{\uparrow}-\Gamma_{15 v}^{\uparrow}$ & 0.74 & 1.71 \\
$E_{g}(\downarrow): \Gamma_{1 c}^{\downarrow}-\Gamma_{15 v}^{\downarrow}$ & 4.0 & 4.5 \\
$\bar{E}_{d_{e g}}(\uparrow)$ & -3.7 & -3.4 \\
$\bar{E}_{d_{t_{2 g}}}(\uparrow)$ & -3.9 & -3.6 \\
$\Delta_{e_{g}}$ & 4.3 & 6.2 \\
$\Delta_{t_{2 g}}$ & 4.8 & 6.7 \\
\hline
\end{tabular}

Table 2 Energy levels and exchange splittings for $\mathrm{ZB}-\mathrm{MnTe}$ in the AF1 phase. All values in eV.

\begin{tabular}{lrrr}
\hline & AF1-LDA & AF1-GW & Expt \\
\hline$E_{g}(\Gamma)$ & 1.37 & 2.7 & $3.2^{a}$ \\
$\bar{E}_{d_{e_{g}}}(\uparrow)$ & -2.2 & -2.2 & \\
$\bar{E}_{d_{t_{2 g}}}(\uparrow)$ & -2.7 & -2.7 & \\
$\bar{E}_{d}(\uparrow)$ & -2.5 & -2.5 & $-3.5^{b}$ \\
$\Delta_{e_{g}}$ & 3.9 & 5.4 & \\
$\Delta_{t_{2 g}}$ & 4.7 & 6.2 & \\
$U$ & 4.4 & 5.9 & $6.9^{c}$ \\
\hline$a-$ Ref.[28, 29][30], $b$-Ref.[16, 17], $c$-Ref.[18]
\end{tabular}

\section{Conclusions}

Our analysis of the electronic structure of zinc-blende MnTe has shown up substantial defects of the LSDA which, at least partially, can be improved within the GW approach: Energy gaps are strongly improved within the GW approximation. However, similarly to non-magnetic II-VI compounds, they are still smaller than in the experiment. A similar effect has been observed for the average exchange splitting of the $\mathrm{Mn}-3 d$ 
states (Hubbard- $U$ ): While there is a correction by $1.5 \mathrm{eV}$ with respect to the LSDA result, the splitting still turns out to be about $1 \mathrm{eV}$ smaller than in the experiment. The binding energy of the Mn- $3 d$ states remains the same within GW as compared to LSDA and is $\sim 1 \mathrm{eV}$ higher than in the experiment. We attribute this fact to the strongly overestimated $p$ - $d$ hybridization in the LDA theory which is not corrected within the GW approach if applied perturbatively. GW predicts the $N_{0} \beta$ parameter close to the corresponding experimental value. On the other hand, there is no improvement with respect to $N_{0} \alpha$. Concluding, the analysis shows that a non self-consistent GW approach is inappropriate to correctly include the important $p$ - $d$ hybridization effects. In our opinion, it is mainly the LDA starting point that needs to be improved. This would be a necessary step for a reliable description of the thermodynamics of DMS materials derived within a model for the local electronic structure taken from ab initio calculations.

Acknowledgements We would like to thank W. Szuszkiewicz for valuable discussions. The numerical calculations have been performed at the ZAM Jülich. Financial support of this work by the Deutsche Forschungsgemeinschaft within the Sonderforschungsbereich 410 is gratefully acknowledged.

\section{References}

[1] J. K. Furdyna, J. Appl. Phys. 64, R29 (1988).

[2] T. Dietl, H. Ohno, F. Matsukura, J. Cibert, D. Ferrand, Science, Vol. 287, 1019 (2000); T. Dietl, Semicond. Sci. Technol. 17, 377 (2002).

[3] B. E. Larson, K. C. Haas, H. Ehrenreich, A. E. Carlsson, Phys. Rev. B 37, 4137 (1988).

[4] P. Hohenberg and W. Kohn, Phys. Rev. 136, B 864 (1965); W. Kohn and L. J. Sham, Phys. Rev. 140, A 1133 (1965).

[5] S.-H. Wei and A. Zunger, Phys. Rev. B 37, 8958 (1988).

[6] U. von Barth and L. Hedin, J. Phys. C 5, 1629 (1972).

[7] L. Hedin and S. Lundqvist, in Solid State Physics, ed. by H. Ehrenreich, F. Seitz, and D. Turnbull, (Academic, New York, 1969), Vol. 23, p. 1.

[8] W. G. Aulbur, L. Jönsson, and J. W. Wilkins, in Solid State Physics, ed. by H. Ehrenreich and F. Spaepen, (Academic, New York, 2000), Vol. 54, p. 1.

[9] A. Fleszar, W. Hanke, Phys. Rev. B 71, 045207 (2005).

[10] A. Fleszar, W. Hanke, Phys. Rev. B 56, 10228 (1997); Phys. Rev. B 62, 2466 (2000).

[11] A. Fleszar, Phys. Rev. B 64, 245204 (2001).

[12] B. Henion, W. Szuszkiewicz, E. Dynowska, E. Janik, T. Wojtowicz, Phys. Rev. B 66, 224426 (2002).

[13] T. M. Giebultowicz, P. Kłosowski, N. Samarth, H. Luo, J. K. Furdyna, J. J. Rhyne, Phys. Rev. B 48, 12817 (1993).

[14] P. W. Anderson, Phys. Rev. 79, 350 (1950); ibid 115, 2 (1959)

[15] P. Djemia, Y. Roussigné, A. Stashkevich, W. Szuszkiewicz, N. Gonzalez Szwacki, E. Dynowska, E. Janik, B. J. Kowalski, G. Karczewski, P. Bogusławski, M. Jouanne and J. F. Morhange, Acta Phys. Pol. A, 106, 239 (2004).

[16] B. J. Kowalski, E. Guziewicz, B. A. Orłowski, E. Janik, G. Karczewski, T. Wojtowicz, J. Kossut, T. van Gemmeren, T. Buslaps and R. L. Johnson, Thin Solid Films 267, 69 (1995).

[17] B. J. Kowalski, B. A. Orłowski, E. Janik, R. L. Johnson, J. Alloys and Comp. 328, 149 (2001).

[18] K. Mimura, H. Sato, S. Senba, H. Namatame, M. Taniguchi, Physica B, 237-238, 392 (1997).

[19] S.-H. Wei and A. Zunger, Phys. Rev. B 35, 2340 (1987).

[20] S.-H. Wei and A. Zunger, Phys. Rev. B 48, 6111 (1993).

[21] N. Gonzalez Szwacki, E. Przeździecka, E. Dynowska, P. Bogusławski and J. Kossut, Acta Phys. Pol. A, 106, 233 (2004).

[22] J. P. Perdew, A. Zunger, Phys. Rev. B 23, 5048 (1981).

[23] D. R. Hamann, Phys. Rev. B 40, 2980 (1989).

[24] G. B. Bachelet, D. R. Hamann, and M. Schlüter, Phys. Rev. B 26, 4199 (1982).

[25] M. Rohlfing, P. Krüger, J. Pollmann, Phys. Rev. Lett. 75, 3489 (1995); Phys. Rev. B 57, 6485 (1998).

[26] S. G. Louie, S. Froyen, and M. L. Cohen, Phys. Rev. B26, 1738 (1982).

[27] H. D. Monkhorst and J. D. Pack, Phys. Rev. B 13, 5188 (1976).

[28] R. Bücker, H.-E. Gumlich and M. Krause, J. Phys. C: Solid State Phys., 18, 661 (1985).

[29] R. L. Gunshor, A. V. Nurmikko, L. A. Kołodziejski, M. Kobayashi and N. Otsuka, J. Crystal Growth 101, 14 (1990).

[30] H.-C. Mertins, H.-E. Gumlich and Ch. Jung, Semicond. Sci. Technol. 8, 1634 (1993).

[31] J. R. Schrieffer, P. A. Wolff, Phys. Rev. 149, 491 (1966).

[32] P. W. Anderson, Phys. Rev. 124, 41 (1961).

[33] J. A. Gaj, R. Planel, G. Fishman, Solid State Commun. 29, 435 (1979). 